\title{
Effect of Delivery Modes on Hearing Screening Results
}

\author{
Ahmet Hamdi Kepekci ${ }^{1,2 *}$ and Ali Bestemi Kepekci ${ }^{3,4}$ \\ ${ }^{1}$ Department of Audiometry, Istanbul Yeni Yuzyil University, Vocational Health High School, Turkey \\ ${ }^{2}$ Department of Otolaryngology, Meltem Hospital, Turkey \\ ${ }^{3}$ Department of Anesthesia, Istanbul Yeni Yuzyil University, Vocational Health High School, Turkey \\ ${ }^{4}$ Department of Anesthesiology and Reanimation, Meltem Hospital, Turkey
}

Submission: August 07, 2019; Published: August 23, 2019

*Corresponding author: Ahmet Hamdi Kepekci, Department of Audiometry, Istanbul Yeni Yuzyil University, Vocational Health High School, Turkey

\begin{abstract}
Background/Aim: The national hearing screening program is applied in newborns. It is reported that the type of anesthesia applied to mothers during delivery may affect hearing screening tests in newborns. In this study, we investigated whether maternal demographic characteristics, type of anesthesia applied to mother, and type of delivery are related to hearing screening test results in newborns.

Materials and Methods: A cross-sectional study of hearing screening tests performed between 01.05.2017-30.04.2019 within the scope of the national newborn hearing screening program, which was born in İstanbul Meltem Hospital, was conducted. Retrospectively, hearing screening data of a total of 2452 newborns were included in the study.

Results: 1977 (80.63\%) of the newborns were successful in the first otoacoustic emission test phase. With the addition of those who passed the second otoacoustic emission test phase, this number rose to 2389 (97.43\%). After the auditory brain response test, the number of those who passed the hearing screening test increased to 2447 (99.80\%) in infants. There was no statistically significant difference between neonatal hearing screening test results and mothers' birth age, gestational week, anesthesia types, and sex of babies ( $p>0.05$ ). The no pass rate of TOAE1 is higher in cesarean section than vaginal delivery. This results improved significantly with the second emission test after 1 week. Detailed information should be given to avoid unnecessary distress in the families of newborns who fail at the first test. The timing of the screening protocol used in neonates should be reassessed.
\end{abstract}

Keywords: Delivery Mode; National Newborn Hearing Screening; Transient Evoked Otoacoustic Emissions; Type of Maternal Anesthesia

\section{Introduction}

The incidence of congenital hearing loss is between 1 and 6 per 1000 live births [1]. Further studies have shown that cognitive and academic deficits in babies with hearing loss will cause secondary behavioral adjustment problems [2,3]. National hearing screening programs were first launched in the United States in March 1993 [4]. In 1994, the first newborn hearing screening started at Marmara University in Turkey. National, The National Newborn Hearing Screening, was initiated in 2004. The screening aims to ensure that all newborns are subjected to the transient evoked otoacoustic emissions (TEOAE) within the first 72 hours [5]. The World Health Organization and the Hearing Loss Committee recommend that rehabilitation should be initiated within the first six months after drawing attention to the first 3 months after birth for the detection of hearing the loss in children [6]. These tests are non-invasive and objective tests. It has been shown that children with hearing loss are detected within the first 3 months and if the necessary rehabilitation is applied before 6 months, the expressive language tests of these children at the age of 3 are found to be within normal limits [7]. The National Hearing Screening Program uses TEOAE and Auditory Brainstem Response (ABR) tests in Newborns [8]. Newborns do not pass these tests can be referred to the upper center. False-positive results are sometimes obtained in these tests. False-positive results require repeated hearing screening tests. In infants who do not pass the TOAE test, the test is repeated after a while. ABR test is performed in infants who do not pass these tests.

This creates anxiety and stress in the baby family. Therefore, it is very important to isolate the factors that may affect the results of newborn hearing screening programs [9]. Cesarean section (CS) saves lives, but CS rates above the $15 \%$ ceiling recommended by the World Health Organization (WHO) in the most developed 


\section{Global Journal of Otolaryngology}

countries appear to be remarkable [10]. The OECD (Organization for Economic Cooperation and Development) report reports that the CS rate increased from $14.4 \%$ in 1990 to $25.8 \%$ in 2009 [11]. In addition to increases in obstetric risk factors, including those associated with delayed and multiple infant pregnancies and maternal obesity, the increase in CS is higher [12]. Studies have shown that CS rates are affected by biological, genetic, and medical factors [13]. It has been reported in the literature that CS lead to cognitive problems of children [14]. Mothers can be anesthetized both during cesarean section and during delivery. There are reports that the type of anesthesia applied to mothers may affect hearing screening tests in newborns $[9,15]$. It is very important to isolate and categorize factors that may affect the success or failure of hearing screening programs. In this study, we investigated whether maternal demographic characteristics, type of anesthesia applied to the mother, and type of delivery were related to hearing screening test results in newborns.

\section{Materials and Methods}

This study is a cross-sectional study of hearing screening tests performed within the scope of National Hearing Screening Program of newborns born in Istanbul Meltem Hospital Table 1: The risk factors defined by the American Academy of Pediatrics Infant Hearing Committee (1994).

\begin{tabular}{|c|}
\hline Risk factors \\
\hline Newborn mothers with TORCH infection (toxoplasmosis, rubella, cytomegalovirus, herpes, syphilis) \\
\hline Family with a history of childhood sensory hearing loss \\
\hline Newborns with the congenital external ear canal and auricle anomalies \\
\hline Premature newborns born with a birth weight below 1500 grams \\
\hline Neonates with high bilirubin levels requiring exchange transfusion \\
\hline Babies of mothers who used ototoxic drugs during pregnancy (e.g. aminoglycosides) \\
\hline Bacterial meningitis \\
\hline Newborns with Apgar score $<5$ in the first minute or $<7$ in the fifth minute \\
\hline Newborns with mechanical ventilation for more than five days \\
\hline Newborns with symptoms related to a syndrome known to accompany hearing loss \\
\hline
\end{tabular}

If amniotic fluid or vernix caseosa accumulates in the external auditory canals, a catheter with a diameter of the canal was inserted into the external auditory canal, and the screening tests were performed. For TOAE and ABR tests, Madsen AccuScreen (GN Otometrics A / S, Taastrup, Denmark) was used. The tests were performed based on the screening protocol valid at the time the study data were collected. The first transient evoked otoacoustic test (TOAE1) was performed on the first day after delivery before the newborn was discharged. Those who did not pass this test were invited to the second emission test (TOAE2) one week later; Those who did not pass TOAE2 were invited to $\mathrm{ABR}$ test 10 days later. Babies who failed the ABR test were referred to a tertiary care center for further hearing tests. If babies born in our hospital are born with vaginal delivery (VD), 24 hours after delivery; if they were born by CS, 48 hours after delivery, they were directed to the Neonatal hearing screening unit to perform TOAE1. Therefore, all TOAE1 tests were between 01.05.2017-30.04.2019. As it was a retrospective study, informed consent was not obtained from the patient, and local ethics committee permission was obtained (Date: 31.05.2019 No: 2019/37). Infants having any of the risk factors identified by the Joint Committee on Infant Hearing of the American Academy of Pediatrics in 1994 (Table 1); infants who remain in the intensive care unit with or without mechanical ventilation after delivery; Infants born before $<35$ weeks of gestation; babies with diagnosed congenital health problems; infants with preeclampsia or eclampsia during pregnancy in the mother were excluded from the study. In 1994, the risk factors defined by the American Academy of Pediatrics Infant Hearing Committee (Items were taken from the Joint Committee on Infant Hearing 1994 Position statement) and were tabulated [16] (Table 1). Neonatal hearing screening tests were performed by experienced audiometrist in a quiet environment in the neonatal hearing screening unit of the hospital. All tests were performed by the same audiometrist. Care was taken to keep the babies quiet and put them on a stretcher during screening. When screening tests were performed in our hospital, the risk factors listed in Table 1 were questioned and recorded in the file of all infants. performed within the first 48 hours after birth. A total of 3105 newborns were screened. Infants with any exclusion criteria in the hearing screening files were excluded from the study. Hearing screening data of a total of 2452 babies were included in the study. Birth types and newborn gender; Age of mothers and gestational weeks are listed. The babies were divided into two groups as VD and CS. The ages of the mothers, gestational weeks, types of anesthesia (those born with VD, those who had epidural anesthesia and delivered by CS, those who delivered by CS, spinal anesthesia, general anesthesia) and newborns were analyzed separately.

\section{Statistical Analysis}

Statistical analysis of the data was performed using IBM SPSS for Windows 23.0 (USA) software, and statistical significance was accepted as $\mathrm{p}<0.05$. Chi-square test was used for statistical evaluation. 


\section{Global Journal of Otolaryngology}

\section{Results}

Of the 2.452 babies, 919 (37.48\%) were born by VD, and 1533 (62.52\%) were born by CS. 1977 (80.63\%) of the newborns passed the screening test at the TOAE1 stage. With the addition of those passing the TOAE2, this number rose to 2389 (97.43\%). The number of babies who passed the hearing tests increased to 2447 (99.80\%) after the ABR test in babies who did not pass the transient evoked otoacoustic tests. Hearing loss was suspected in $5(0.20 \%)$ babies, and these babies were referred to a tertiary health care facility. When the analysis of the tests was done according to the type of birth, the results were as shown in Table 2. $87.27 \%(802 / 919)$ of babies born with VD passed the TOAE1 test. The ratio increased to $97.93 \%$ (900/919) with the addition of TOAE2 testers. The ratio increased to $97.93 \%$ (900/919) with the addition of TOAE2 testers. The rate of passing the test was 99.78\% (917/919) after ABR testing of newborns from TOAE2. As a result, only $0.22 \%(2 / 919)$ of the babies born with VD were transferred to the tertiary health care facility from all tests. $76.65 \%$ (1175/1533) of babies born with CS passed TOAE1 test. The ratio increased to $97.13 \%(1489 / 1533)$ with the addition of the TOAE2. After the ABR test, the pass rate increased to $99.80 \%$ (1530/1533). As a result, only $0.20 \%(3 / 1533)$ of babies born by CS were passed from all tests and referred to a tertiary health care facility.

Table 2: Newborn hearing screening test results by delivery of mode.

\begin{tabular}{|c|c|c|c|c|c|c|c|}
\hline \multirow{3}{*}{$\begin{array}{c}\text { Newborn } \\
\text { Hearing } \\
\text { Screening } \\
\text { Test }\end{array}$} & \multirow{3}{*}{ Total } & \multirow[b]{3}{*}{ Fail } & \multicolumn{3}{|c|}{ Delivery Mode } & & \multirow{3}{*}{ p value } \\
\hline & & & \multicolumn{2}{|c|}{ VD } & \multicolumn{2}{|c|}{ CS } & \\
\hline & & & Pass & Fail & Pass & Fail & \\
\hline TOAE1 & 1977 (80.63\%) & 475 (19.37\%) & $802(87.27 \%)$ & 117 (12.73\%) & 1175 (76.65\%) & $358(23.35 \%)$ & 0.00 \\
\hline TOAE2 & $412(97.43 \%)$ & $63(2.57 \%)$ & 98 (97.93\%) & $19(20.07 \%)$ & 314 (97.13\%) & $44(2.87 \%)$ & 0.27 \\
\hline ABR & $58(99.80 \%)$ & $5(0.20 \%)$ & 17 (99.78\%) & $2(0.22 \%)$ & 41 (99.80\%) & $3(0.20 \%)$ & 0.61 \\
\hline
\end{tabular}

Abbreviations: TOAE1: The first transient evoked otoacoustic test; TOAE2: The second transient evoked otoacoustic test; ABR: Auditory brainstem response; CS: Cesarean section; VD: Vaginal delivery

The difference between the number of newborns remaining in the TOAE1 stage between the two groups was statistically significant $(\mathrm{p}<0.05)$. After adding the number of testers in TOAE2 stage, no statistically significant difference was found between the two groups ( $p>0.05)$. No statistically significant difference was found between the two groups which were referred to the third step (i.e., all the tests) ( $p>0.05$ ). Of the 2 babies who were born with VD, 1 failed to screen in 1 ear and 1 failed in 2 ears. Of the 3 babies who were born with CS, 1 failed to screen in 1 ear and 2 failed in 2 ears. False-positive rates were high $(12.73 \%$ $23.35 \%$ ) in the first hearing screening in both delivery types and significantly decreased in the second screening (2.07\% - 2.87\%) (Figure 1). Accordingly, it was observed that most of the babies passed the screening test at TOAE2 stage, and there was no statistically significant difference in terms of delivery type ( $\mathrm{p}=$ 0.417 ). There was no statistically significant difference between the mothers' age at birth, gestational week, anesthesia types and sex of babies and hearing test results ( $p>0.05)$.

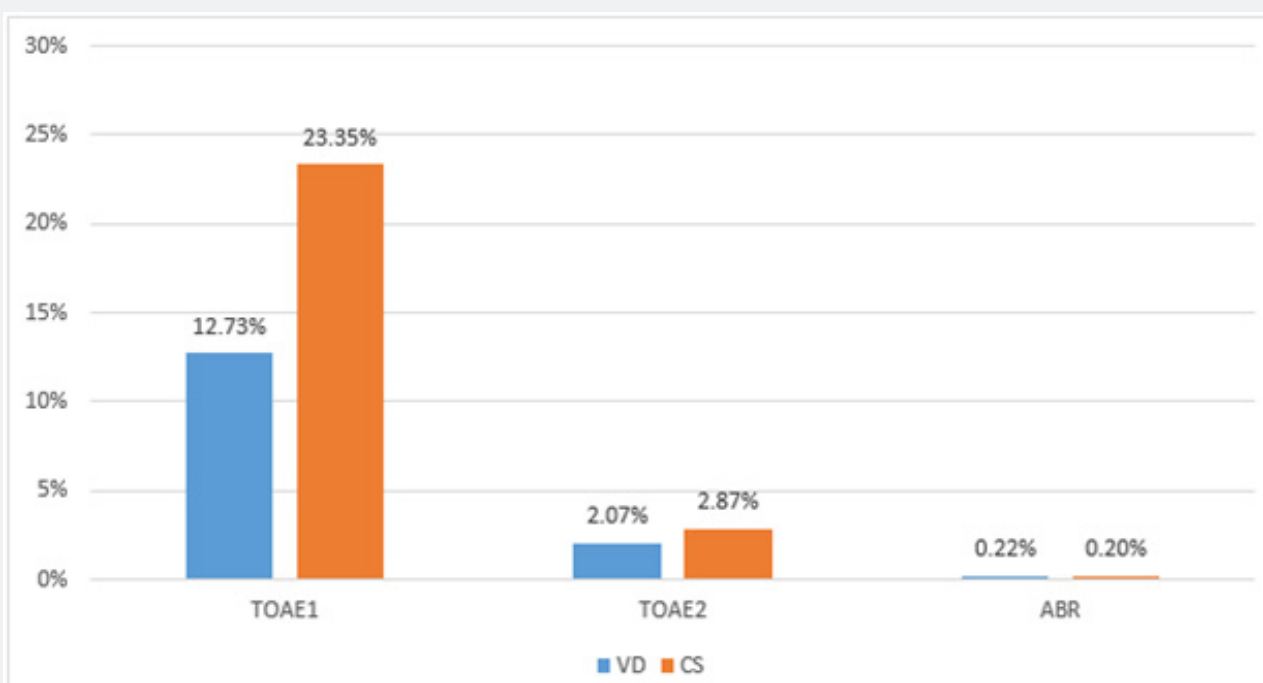

Figure 1: Distribution of infants that do not pass by neonatal hearing screening tests according to delivery modes. Abbreviations: TOAE1: The first transient evoked otoacoustic test; TOAE2: The second transient evoked otoacoustic test; ABR: Auditory brainstem response; CS: Cesarean section; VD: Vaginal delivery 


\section{Discussion}

Hearing screening program is the method that can detect early hearing loss in newborns. Tests to be used in screening programs should be interpreted as easily and quickly as possible. TOAE and ABR tests performed within the framework of the national hearing screening program are non-invasive and easily reproducible tests [16]. Determining factors that may affect screening test results will lead to the development of these programs. In this study, we compared the results of neonatal hearing screening with the factors related to mother and baby. Failure of TOAE1 was higher in newborns born by CS. In both delivery modes, it was seen that the majority of newborns completed the test at the TOAE2 stage. Almost all of those who failed in TOAE2 completed the screening in the ABR phase. After all stages, no correlation was found between hearing screening test results and mode of delivery. Smolkin et al. [17] reported that the risk of failure of TOAE1 in infants born by CS increased approximately 3.2 times compared with VD. In this paper, the result was associated with amniotic fluid accumulation in the middle ear [17]. In a similar study, Xiao at all. found significantly higher failure rates in CS in TOEA1. They suggested that this difference could be seen in babies born by CS due to delayed absorption of middle ear fluid [18]. They grouped the results of TOAE1 according to screening timing, indicating that those patients screened in the first 42 hours showed even higher failures than those screened at later hours. However, the rates they found were not statistically significant [18].

Olusanya [19] in their study on Nigerian newborns born with VD compared to those born with CS, they are two times the risk of hearing loss, he said. As a result, they attributed Nigerian pregnant women to increase the risk of birth trauma by spending more than necessary labor for VD. In the same study, babies with hearing loss were associated with low Apgar scores [19]. Farahani et al. [20] It has been reported that hearing loss results after hearing screening tests are 1.5 times higher in VD than those performed by CS. The authors attributed this difference to incorrect tympanic membrane mobility in infants born by VD, thereby delaying the absorption of middle ear fluid. He stated that false-positive rates decreased in time with CS but did not see such a decrease in those born with VD [20]. In the same publication, they reported that false-positive rates decreased significantly in TOAE2 tests performed two weeks after birth and that there was no significant difference in terms of delivery mode [20]. In the study of Güven, it was stated that those who delivered by CS were more unsuccessful in TOAE1, but the rate of failure decreased as the hour passed in both delivery modes [21]. In the same publication, tests performed after the 60 th hour of labor were compared, and it was stated that there was no significant difference between failure rates in terms of delivery modes over time [21]. In another study, Smokin et al. [19] emphasized that performing TOAE1 screening 48 hours after birth will reduce the failure rates [22].
In our study, it was found that failure rates in TOAE1 decreased in both forms from birth to screening time. In infants who failed the TOAE1 test, otoacoustic emission tests were repeated before discharge. The results of the second screening showed low failure rates over time. However, failure rates after TOAE1 were higher in CS. Failure rate after CS after TOEA1 was $23.35 \%$ and $12.73 \%$ for VD. The difference was statistically significant. TOAE2 results, ABR test results, and rates of infants with hearing loss referred to a tertiary care facility were similar in both delivery groups. After the ABR tests, the number of infants who did not pass the hearing screening test decreased significantly. Because failure rates in TOAE1 have declined over time and TOAE2 results are similar in the two groups, we think this is probably due to low tympanic membrane mobility in the middle ear or amniotic fluid accumulation in the early postnatal period. We believe that the failure rate of TOAE1 is higher in $\mathrm{CS}$, and tympanic membrane motility is better in VD. As time passes, the amniotic fluid is absorbed. Accordingly, the TOAE2 test performed after 1 week shows that the failure rates of both births are close to each other. Sequi-Canet [23] results are similar to our results.

These authors found that babies born with VD were more successful in TOAE1 with early onset of breastfeeding. Torrico et al. [24] recommended that it should be done as late as possible 48 hours after birth in order to increase the success rate in TOAE1 test and recommended that repeat should be done at least six days after the first test in case of failure. The data analyzed in this study show that the delivery type does not affect the success of newborn hearing screening tests. However, TOAE1 results are related to the way newborns are delivered. Possible amniotic fluid in the outer ear and middle ear may affect the success of TOAE1 screening in the early postnatal period. In one study, it was reported that the removal of external ear rash increased the rate of TOAE transition from $76 \%$ to $91 \%$ [25]. Cavanaugh et al. [26] Reported that tympanic membrane mobility was limited in most newborns and that $93 \%$ had normal tympanic membrane appearance (pink or gray color) in most neonates. Diaz et al. [27] showed that maternal lidocaine hydrochloride anesthesia produced a significant delay in brainstem auditory evoked responses in newborns born by CS. Khoza et al. [28] stated that epidural anesthesia applied four hours before birth increased the frequency of false-positive results in hearing screening tests.

In our study, no relationship was found between anesthesia types applied to mothers in VD and CS and hearing screening test results of newborns. Epidural anesthesia for mothers in VD in our hospital is rare; the fact that the majority of CS was performed with spinal anesthesia may have been effective in this. In this study, false-positive results in TOAE1 are common, with a higher rate in babies born by CS. It should be remembered that false-positive results may cause anxiety and stress in the family. Families should be well informed about this, and it should be stated that the important result will be said after the completion of all tests. Care should be taken to ensure that screening tests 
are performed properly and on time. Infants who fail hearing screening tests should be referred to as tertiary health care facilities without delay. The timing of TOAE1, where falsepositive results are frequently encountered, should be planned as late as possible just before the baby is discharged.

\section{Conclusion}

The type of delivery in newborns and the type of anesthesia applied to their mothers do not affect hearing screening test results. The failure rate of the first otoacoustic emission tests is higher in cesarean-born infants. This result, which was evaluated as false-positivity, was significantly improved by the second transient evoked otoacoustic test after 1 week. In particular, the families of newborns who failed the first transient evoked otoacoustic test should be given detailed information to avoid unnecessary sadness. Furthermore, the timing of the screening protocol used in neonates should be reassessed.

\section{References}

1. Cunningham M (2003) Cox e, Committee on Practice and Ambulatory Medicine and the Section on Otolaryngology and bronchoesophagology Hearing assessment in infants and children: recommendations beyond neonatal screening. Pediatrics 111: 436-440.

2. Fitzpatrick EM, Durieux-Smith A, Whittingham J (2010) Clinical practice for children with mild bilateral and unilateral hearing loss. Ear and hearing 31(3): 392-400.

3. Culbertson JL, Gilbert LE (1986) Children with unilateral sensorineural hearing loss: cognitive, academic, and social development. Ear and hearing $7(1)$ : 38-42.

4. WHO (2009) Newborn infant hearing screening: Current issues and guiding principles for action? WHO Library.

5. Arslan S, Ișik A, Imamoğlu M, Topbaș M, Aslan Y, et al. (2013) Universal newborn hearing screening; automated transient evoked otoacoustic emissions. B-ENT 9(2): 122-131.

6. Hearing J CoI (2007) Joint Committee on Infant Hearing year 2007 position statement for early detection and intervention programs. Pediatrics 120: 898-921.

7. Yoon PJ, Price M, Gallagher K, Fleisher BE, Messner AH (2003) The need for long-term audiologic follow-up of neonatal intensive care unit (NICU) graduates. Int J Pediatr Otorhinolaryngol 67(4): 353-357.

8. Kepekçi AH (2018) Section 2: Sub-Fields in Audiometry. Audiometry: Hearing and Measurement Vestibular System, Neurotology: Nobel Medical Bookstores pp. 105-124.

9. Gibbons L, Belizán JM, Lauer JA, Betrán AP, Merialdi M, et al. (2010) The global numbers and costs of additionally needed and unnecessary caesarean sections performed per year: overuse as a barrier to universal coverage. World health report 30: 1-31.

10. Indicators O (2015) Health at a Glance 2011. OECD Indicators, OECD Publishing, Paris.

11. Stavrou EP, Ford JB, Shand AW, Morris JM, Roberts CL (2011) Epidemiology and trends for Caesarean section births in New South Wales, Australia: a population-based study. BMC pregnancy and childbirth 11: 8 .
12. Santas G, Santas F (2018) Trends of caesarean section rates in Turkey. Journal of Obstetrics and Gynaecology 38(5): 658-662.

13. Sundet JM, Tambs K, Harris JR, Magnus P, Torjussen TM (2005) Resolving the genetic and environmental sources of the correlation between height and intelligence: A study of nearly 2600 Norwegian male twin pairs. Twin Research and Human Genetics 8(4): 307-311.

14. Gurian EA, Kinnamon DD, Henry JJ, Waisbren SE (2006) Expanded newborn screening for biochemical disorders: the effect of a falsepositive result. Pediatrics 117(6): 1915-1921.

15. Hearing J CoI (1995) Joint committee on infant hearing 1994 position statement. Pediatrics 95(1): 152-156.

16. Paludetti G, Ottaviani F, Fetoni A, Zuppa A, Tortorolo G (1999) Transient evoked otoacoustic emissions (TEOAEs) in new-borns: normative data International journal of pediatric otorhinolaryngology 47(3): 235-241.

17. Smolkin T, Mick O, Dabbah M, Blazer S, Grakovsky G, et al. (2012) Birth by cesarean delivery and failure on first otoacoustic emissions hearing test. Pediatrics 130(1): e95-e100.

18. Xiao T, Li Y, Xiao L, Jiang L, Hu Q (2015) Association between mode of delivery and failure of neonatal acoustic emission test: a retrospective analysis. International journal of pediatric otorhinolaryngology 79(4): 516-519.

19. Olusanya BO, Solanke OA (2009) Maternal and neonatal factors associated with mode of delivery under a universal newborn hearing screening programme in Lagos, Nigeria. BMC pregnancy and childbirth 9: 41 .

20. Farahani F, Nahrani MH, Seifrabiei MA, Emadi M (2017) The effect of mode of delivery and hospital type on newborn hearing screening results using otoacoustic emissions: based on screening age. Indian Journal of Otolaryngology and Head \& Neck Surgery 69: 1-5.

21. Güven SG (2019) The Effect of Mode of Delivery on Newborn Hearing Screening Results. Turkish archives of otorhinolaryngology 57: 19.

22. Smolkin T, Awawdeh S, Blazer S, Mick O, Makhoul IR (2013) Delayed first otoacoustic emissions test decreases failure on neonatal hearing screening after caesarean delivery. Acta Paediatrica 102: e194-e199.

23. Sequi-Canet JM, Sala-Langa MJ, del Castillo JIC (2014) Perinatal factors affecting the detection of otoacoustic emissions in vaginally delivered, healthy newborns, during the first 48 hours of life. Acta Otorrinolaringologica 65: 1-7.

24. Torrico P, Gómez C, López-Ríos J, de Caceres MC, Trinidad G, et al. (2004) Age influence in otoacoustic emissions for hearing loss screening in infants. Acta otorrinolaringologica espanola 55(4): 153-159.

25. Chang KW, Vohr BR, Norton SJ, Lekas MD (1993) External and middle ear status related to evoked otoacoustic emission in neonates. Archives of Otolaryngology-Head \& Neck Surgery 119(3): 276-282.

26. Cavanaugh RM (1987) Pneumatic otoscopy in healthy full-term infants. Pediatrics 79(4): 520-523.

27. Diaz M, Graff M, Hiatt IM, Friedman S, Ostfeld B, et al. (1988) Prenatal lidocaine and the auditory evoked responses in term infants. Am J Dis Child 142(2): 160-161.

28. Khoza-Shangase K, Joubert K (2011) The influence of epidural anesthesia on new-born hearing screening: A pilot study. J Pharm Bio allied Sci 3(1): 135-141. 


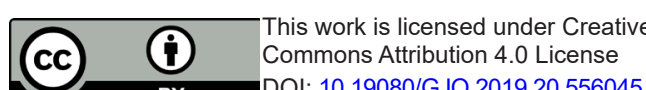

BY DOI: 10.19080/GJO.2019.20.556045
Your next submission with Juniper Publishers will reach you the below assets

- Quality Editorial service

- Swift Peer Review

- Reprints availability

- E-prints Service

- Manuscript Podcast for convenient understanding

- Global attainment for your research

- Manuscript accessibility in different formats

( Pdf, E-pub, Full Text, Audio)

- Unceasing customer service

Track the below URL for one-step submission https://juniperpublishers.com/online-submission.php 